\title{
Tailored tungsten lattice structures for plasma-facing components in magnetic confinement fusion devices
}

\author{
A. v. Müller ${ }^{1,2}$, M. Binder ${ }^{3}$, G. Calabro ${ }^{4}$, R. De Luca ${ }^{4}$, P. Fanelli $^{4}$, \\ R. Neu ${ }^{1,2}$, G. Schlick ${ }^{3}$, F. Vivio ${ }^{5}$, J.H. You ${ }^{1}$ \\ ${ }^{1}$ Max-Planck-Institut für Plasmaphysik, 85748 Garching, Germany \\ ${ }^{2}$ Technische Universität München, 85748 Garching, Germany \\ ${ }^{3}$ Fraunhofer IGCV, 86159 Augsburg, Germany \\ ${ }^{4}$ University of Tuscia, 01100 Viterbo, Italy \\ ${ }^{5}$ Tor Vergata University of Rome, 00133 Rome, Italy
}

Nuclear fusion is known to be an energy source that holds the potential to provide a sustainable, environmentally responsible and dispatchable high power density energy supply solution for the future of mankind. The most promising approaches to harness fusion energy are nowadays based on magnetically confined high-temperature plasmas within specifically designed toroidal devices [1]. Persistent research on thermonuclear magnetic confinement fusion has led to current design activities regarding a demonstration fusion reactor (DEMO) which is foreseen to be realised as a so-called tokamak type reactor [2]. One predominantly challenging issue with respect to the realisation of a DEMO reactor is the design and manufacture of highly loaded plasma-facing components (PFCs) that have to sustain intense particle, heat and neutron fluxes during fusion operation [3]. For such PFCs, specific high performance materials are needed in order that reliable components can be designed. Regarding materials that are directly facing a fusion plasma, tungsten (W) is currently considered the preferred plasma-facing material (PFM) for future magnetic confinement thermonuclear fusion reactors. This is mainly due to the fact that $\mathrm{W}$ exhibits a high threshold energy for sputtering by as well as a low retention of hydrogen isotopes which represent the fuel for the fusion reactions [4]. A particularly critical aspect with respect to PFCs in a DEMO reactor are transient wall loadings that can e.g. arise due to plasma instabilities in a tokamak. Such transient events can lead to very intense heat loadings (several tens of $\mathrm{GW} / \mathrm{m}^{2}$ for time periods of a few $\mathrm{ms}$ ) on PFCs that in turn can damage the blanket structures of a reactor severely [5]. In order to protect the wall of a fusion reactor against such events specific limiter PFCs are currently being investigated. These components are foreseen to baffle the short and intense heat pulses to the reactor walls in order that blanket structures behind these limiter components are not thermally overloaded or damaged. A possible material solution for such limiter PFCs is the use of tailored porous W materials. With such metamaterials, components can be realised that combine an overall low thermal conductance due to the incorporated porosity with the beneficial plasma-wall interaction properties of W. However, W is a difficult material to work with as it is an intrinsically hard and brittle metal which means that the machining of $\mathrm{W}$ is laborious and expensive. Against these limitations, additive manufacturing (AM) methods represent a versatile approach for the realisation of geometrically complex $\mathrm{W}$ parts. The characteristic feature of AM processes is that three-dimensional objects are created by sequential layerwise deposition of material under computer control which means that with such an approach parts with high geometrical complexity can be realised straightforwardly. In recent years, substantial progress has been achieved regarding the AM of metals by means of laser powder bed fusion (LPBF) which is a technology that allows the direct AM of a wide variety of metals without the need for binder phases. During LPBF, raw powder material is selectively melted and consolidated by means of a laser beam that is focused onto a powder bed [6]. The cover image illustrates the top view on a W specimen with a tailored lattice architecture that was fabricated by means of LPBF. Such porous $\mathrm{W}$ lattices are currently being investigated with respect to limiter PFCs as described above. The illustrated specimen is a lattice structure that is derived from a parametric solid model based on the repetition of a tetrakaidecahedron (Kelvin model). Such a model was in the past also applied and validated with respect to open-cell Aluminium foams [7]. The parameters for the illustrated W lattice 
structure were derived with the help of a design optimisation yielding a tailored anisotropic lattice geometry that fulfils functional requirements in terms of the thermal diffusivity behaviour. For the fabrication of the illustrated W lattice specimen an Aconity $O N E$ LPBF facility equipped with a fibre laser operating at a wavelength of $1075 \mathrm{~nm}$ was used. The specialty of this facility is an inductive heating system with which high build plate temperatures of up to $1000^{\circ} \mathrm{C}$ can be ensured during the AM process. It is known that such a preheating is beneficial during LPBF processing of high melting point refractory metals with a high ductile-to-brittle transition temperature (DBTT), like W. Regarding laser exposure parameters for LPBF processing of pure $\mathrm{W}$ on the abovementioned facility previous studies showed that energy densities of about $250 \mathrm{~J} / \mathrm{mm}^{3}$ yield good consolidation of approximately $98 \%$ relative mass density when spheroidised pure $\mathrm{W}$ powder as well as Argon as protective atmosphere are used [8]. However, $\mathrm{W}$ is a particularly challenging material for LPBF processing due to the intrinsic properties of this metal, especially the high DBTT, in combination with the high thermal gradients that occur during a LPBF process due to the laser-material interaction (spatial temperature gradients of approximately $10^{2} \mathrm{Kmm}^{-1}$ to $10^{4} \mathrm{Kmm}^{-1}$ as well as cooling rates higher than $\left.10^{4} \mathrm{Ks}^{-1}[6]\right)$. Hence, W parts fabricated by means of LPBF typically exhibit microcracks that clearly manifest during metallographic investigations. Up to now, several studies confirm this microcrack formation but there is currently no method known that completely mitigates microcracks in W consolidated by means of LPBF [9-14]. Nevertheless, the illustrated cover image demonstrates that the AM of complex and tailored W structures is feasible. Upcoming research work will comprise the thermal and mechanical characterisation as well as the investigation of plasma-material interaction properties of $\mathrm{W}$ lattice structures as illustrated in the image. These investigations will reveal if such materials exhibit adequate properties that are appropriate with respect to PFC applications in magnetic confinement fusion devices.

\section{Acknowledgements}

This work has been carried out within the framework of the EUROfusion Consortium and has rceived funding from the Euratom research and training programme 2014-2018 and 2019-2020 under grant agreement No. 633053. The views and opinions expressed herein do not necessarily reflect those of the European Commission.

[1] J. Ongena et al., Magnetic-confinement fusion, Nature Physics 12, 398-410 (2016), https://doi.org/10.1038/nphys3745

[2] G. Federici et al., DEMO design activity in Europe: Progress and updates, Fusion Engineering and Design 136 (2018) 729-741, https://doi.org/10.1016/j.fusengdes.2018.04.001

[3] Ch. Linsmeier et al., Development of advanced high heat flux and plasma-facing materials, Nuclear Fusion 57 (2017) 092007, https://doi.org/10.1088/1741-4326/aa6f71

[4] D. Stork et al., Developing structural, high-heat flux and plasma facing materials for a nearterm DEMO fusion power plant: The EU assessment, Journal of Nuclear Materials 455 (2014) 277 291, https://doi.org/10.1016/j.jnucmat.2014.06.014

[5] F. Maviglia et al., Wall protection strategies for DEMO plasma transients, Fusion Engineering and Design 136 (2018) 410-414, https://doi.org/10.1016/j.fusengdes.2018.02.064

[6] T.S. Srivatsan and T.S. Sudarshan, Additive Manufacturing: Innovations, Advances, and Applications, CRC Press, 2016. ISBN 978-1-4987-1478-5.

[7] P. Fanelli et al., Modelling and characterization of structural behaviour of Al open-cell foams, Materials and Design 114, 167-175 (2017), https://doi.org/10.1016/j.matdes.2016.10.052 
[8] A. v. Müller et al., Additive manufacturing of pure tungsten by means of selective laser beam melting with substrate preheating temperatures up to $1000^{\circ} \mathrm{C}$, Nuclear Materials and Energy 19 (2019) 184-188, https://doi.org/10.1016/j.nme.2019.02.034

[9] K. Deprez et al., Rapid additive manufacturing of MR compatible multipinhole collimators with selective laser melting of tungsten powder, Medical Physics 40 (1) (2013) 012501, https://doi.org/10.1118/1.4769122

[10] A. Iveković et al., Selective laser melting of tungsten and tungsten alloys, International Journal of Refractory Metals and Hard Materials 72 (2018) 27-32, https://doi.org/10.1016/j.ijrmhm.2017.12.005

[11] D. Wang et al., Dense pure tungsten fabricated by selective laser melting, Applied Sciences 7 (4) (2017) 430, https://doi.org/10.3390/app7040430

[12] C. Tan et al., Selective laser melting of high-performance pure tungsten: parameter design, densification behavior and mechanical properties, Science and Technology of Advanced Materials 19 (1) (2018) 370-380, https://doi.org/10.1080/14686996.2018.1455154

[13] J. Braun et al., Molybdenum and tungsten manufactured by selective laser melting: Analysis of defect structure and solidification mechanisms, International Journal of Refractory Metals and Hard Materials 84 (2019) 104999, https://doi.org/10.1016/j.ijrmhm.2019.104999

[14] B. Vrancken et al., In-situ characterization of tungsten microcracking in Selective Laser Melting, Procedia CIRP, Volume 74, 2018, Pages 107-110, https://doi.org/10.1016/j.procir.2018.08.050 